\title{
ARTICLE \\ Melatonin receptor agonist ramelteon attenuates mouse acute and chronic ischemic brain injury
}

\author{
Xiao-li Wu' ${ }^{1}$, Shou-sheng Lu' ${ }^{1,2}$, Meng-ru Liu' ${ }^{1}$, Wei-dong Tang ${ }^{1}$, Jun-zi Chen ${ }^{3}$, Yan-rong Zheng ${ }^{1}$, Anil Ahsan ${ }^{1}$, Ming Cao', Lei Jiang ${ }^{1}$, \\ Wei-wei $\mathrm{Hu}^{1}$, Jia-ying $\mathrm{Wu}^{1}$, Zhong Chen ${ }^{1}$ and Xiang-nan Zhang ${ }^{1}$
}

\begin{abstract}
Melatonin receptors (MTs) are potential drug targets for stroke therapy. Ramelteon is a selective melatonin receptor agonist used to treat insomnia. In this study we investigated whether ramelteon could attenuate cerebral ischemia in mice. Acute focal cerebral ischemia was induced in mice via middle cerebral artery occlusion (MCAO). We found oral administration of ramelteon $(3.0 \mathrm{mg} / \mathrm{kg}$ ) significantly attenuated ischemic injury even when it was given $4 \mathrm{~h}$ after the onset of ischemia. We showed that administration of ramelteon $(3.0 \mathrm{mg} / \mathrm{kg})$ displayed comparable protective efficacy and length of effective time window as administration of edaravone (10 mg/kg, i.p.), which was used in clinic to treat ischemic stroke. Chronic ischemic brain injury was induced in mice using photothrombosis. Oral administration of ramelteon $\left(3.0 \mathrm{mg} \cdot \mathrm{kg}^{-1} \cdot \mathrm{d}^{-1}\right)$ for 7 days after ischemia significantly attenuated functional deficits for at least 15 days. The neuroprotection of ramelteon was blocked by 4-P-PDOT, a specific MT antagonist. We further revealed that ramelteon significantly inhibited autophagy in the peri-infarct cortex in both the mouse ischemia models via regulating AMPK/mTOR signaling pathway. Intracerebroventricular injection of rapamycin, an autophagy activator, compromised the neuroprotection of ramelteon, suggesting ramelteon might attenuate ischemic injury by counteracting autophagic cell death. These data demonstrate for the first time the potential benefits of ramelteon in the treatment of both acute and chronic ischemic brain injury and provide the rationale for the application of ramelteon in stroke therapy.
\end{abstract}

Keywords: cerebral ischemia; ramelteon; autophagy; middle cerebral artery occlusion (MCAO); photothrombosis; melatonin receptors

Acta Pharmacologica Sinica (2020) 41:1016-1024; https://doi.org/10.1038/s41401-020-0361-2

\section{INTRODUCTION}

Cerebral ischemia is one of the leading causes of mortality and disability worldwide [1, 2]. The administration of tissue plasminogen activator (tPA) within the therapeutic time window is one of the few available therapies for stroke. However, only a small portion of patients benefit from this thrombolytic treatment [3-5]. After the occlusion of arteries, approaches to increase collateral flow may attenuate the ischemic injury [6]. Therefore, intensive efforts have been made to find therapeutic targets and related drugs for stroke treatment.

Melatonin protects against ischemic brain injury by pleiotropic mechanisms, including anti-oxidation, anti-inflammation, anti-apoptosis and the reduction of autophagic cell death [7-11]. These findings suggest that melatonin can be a novel therapy for ischemic stroke treatment [12]. It has become clear in recent years that melatonin confers its neuroprotection, at least partly, by activating its receptors, namely, MT1 and MT2, in ischemic brains $[13,14]$. MTs activate a variety of signaling cascades rapidly and subsequently trigger the protective effect, i.e., the reduction of ischemia-induced inflammation, oxidative stress and mitochondrial dysfunction [7, 15]. In addition, the recent discoveries that $\mathrm{MT}$ agonists attenuate ischemic brain injury further shed light on MTs as promising drug targets for stroke therapy $[15,16]$.

Ramelteon is an FDA-approved MT1/MT2 agonist used for insomnia. Ramelteon acts selectively on MTs and shows limited affinity to the receptors of other neurotransmitters in the central nervous system, including GABA, serotonin, dopamine, noradrenaline and acetylcholine [17, 18]. Ramelteon has been proven effective in preventing delirium and is safe for long-term use. Emerging studies have shown the cardioprotective effect of ramelteon in reducing ischemic injury, suggesting additional pharmacological activities of ramelteon other than being a sleep agent [19]. However, whether ramelteon can serve as a potential drug to ameliorate ischemic brain injury has never been evaluated.

\section{MATERIALS AND METHODS}

Animals

Male C57BL/6 mice weighing 22-25 g were used. All experiments were approved by and conducted in accordance with the ethical guidelines of the Zhejiang University Animal Experimentation Committee and were in complete compliance with the National Institutes of Health Guide for the Care and Use of Laboratory

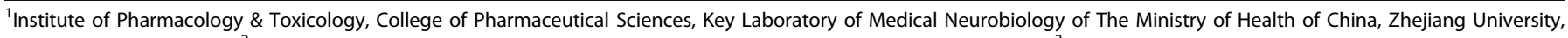
Hangzhou 310058, China; ${ }^{2}$ Department of Pharmacy, First People's Hospital of Linhai City, Linhai 317000, China and ${ }^{3}$ Hangzhou No.4 High school, Hangzhou 310018, China Correspondence: Zhong Chen (chenzhong@zju.edu.cn) or Xiang-nan Zhang (xiangnan_zhang@zju.edu.cn)

These authors contributed equally: Xiao-li Wu, Shou-sheng Lu

Received: 26 September 2019 Accepted: 2 January 2020

Published online: 27 February 2020 
Animals. Efforts were made to minimize any pain or discomfort, and the minimum number of animals was used.

MCAO mouse model and the determination of ischemic injury Focal cerebral ischemia was induced by middle cerebral artery occlusion (MCAO) [20]. In brief, mice were anesthetized by the inhalation of isoflurane for surgery. Cerebral blood flow (CBF) was determined in the area of the middle cerebral artery (MCA) by laser Doppler flowmetry (Model Moor VMS-LDF2, UK). A probe was attached to the skull over the cortex supplied by the right MCA (2$\mathrm{mm}$ caudal to bregma and 6-mm lateral to midline). A 6-0 nylon monofilament suture with a rounded tip was inserted $\sim 10 \mathrm{~mm}$ into the internal carotid to occlude the origin of the MCA. Animals with less than an $80 \%$ reduction in $\mathrm{CBF}$ were excluded from the study. Mice underwent MCAO for $24 \mathrm{~h}$ or $96 \mathrm{~h}$. Body temperature was maintained at $37^{\circ} \mathrm{C}$ until the animals recovered from the anesthesia. To analyze protein expression, the penumbral tissue of ischemic brains was rapidly collected after $24 \mathrm{~h}$ or $96 \mathrm{~h}$ of MCAO, as previously described [21].

Infarct volumes were measured at $24 \mathrm{~h}$ or $96 \mathrm{~h}$ after surgery, and coronal mouse brain slices at 2-mm intervals were stained with $0.25 \%$ TTC (Sigma, T8877). Infarcted areas were analyzed using Image Pro Plus 7.0 and measured by the indirect method, which corrects for edema [20].

Neurological deficit scores were evaluated at $24 \mathrm{~h}$ or $96 \mathrm{~h}$ after surgery as follows: 0 , no deficit; 1 , flexion of the contralateral forelimb upon lifting the whole animal by the tail; 2 , circling to the contralateral side; 3 , falling to the contralateral side; and 4, no spontaneous motor activity [22].

Photothrombotic model of stroke in mice

To induce chronic focal cerebral ischemia, photothrombosis was performed in mice as described previously [23]. Mice were anesthetized with isoflurane and mounted in a stereotaxic apparatus (Stoelting, 512600). A cold light source (diameter $1.8 \mathrm{~mm} ; 12,000$ lux) was placed in close contact with the skull surface at $1.5 \mathrm{~mm}$ lateral from bregma. Rose Bengal solution (Sigma; 330000) was administered intraperitoneally at a dose of $100 \mathrm{mg} / \mathrm{kg}$. Five minutes after injection, the brain was illuminated for $15 \mathrm{~min}$ through the intact skull to initiate photothrombosis. Sham mice were treated with the same procedure without light illumination. For protein analysis, 15 days after photothrombosis, the brain cortex around the infarct core was rapidly dissected as previously described [24].

\section{Drug administration}

Ramelteon (MCE, A0014) was dissolved in saline containing $0.5 \%$ carboxymethylcellulose (CMC). Animals were given ramelteon $(0.3,1.0$ or $3.0 \mathrm{mg} / \mathrm{kg}, i . g$.) at the onset of MCAO. For the treatment after ischemia, ramelteon $(3.0 \mathrm{mg} / \mathrm{kg}, i . g$.) was administered at the onset of or at 4 and $6 \mathrm{~h}$ after MCAO. Edaravone (Sigma, M70800) was intraperitoneally injected at doses of $3,10,30 \mathrm{mg} / \mathrm{kg}$ at the indicated time after MCAO. For the treatment of chronic cerebral ischemia, ramelteon $(3.0 \mathrm{mg} / \mathrm{kg}, i . g$.) was administered daily after photothrombosis for 7 days. The MTs antagonist 4-P-PDOT (Tocris, 1034) was administered $(3.0 \mathrm{mg} / \mathrm{kg}$, i.p.) $0.5 \mathrm{~h}$ before ramelteon administration [25]. Rapamycin (Sigma, R0395) was dissolved in saline and intracerebroventricularly $(2 \mu \mathrm{L}, 10 \mu \mathrm{mol} / \mathrm{L})$ injected at the onset of ischemia.

\section{Grid-walking task}

The grid-walking task apparatus was manufactured, as previously described [23]. The grid area was $32 \mathrm{~cm} \times 20 \mathrm{~cm} \times 50 \mathrm{~cm}$ (length $\times$ width $\times$ height) with a $12 \mathrm{~mm}$ square wire mesh. Mice walked on a wire grid for $3 \mathrm{~min}$ while being video-recorded. Foot faults for the forelimb and the total normal steps were counted. The foot fault index was calculated as the number of foot faults/(foot faults + the number of non-foot-fault steps) $\times 100 \%$. Foot faults were considered to occur when the forelimbs passed through the grid hole. The video analysis was performed by individuals who were blinded to these experiments.

\section{Cylinder task}

Mice were placed in a Plexiglas cylinder $(15 \mathrm{~cm}$ in height with a diameter of $10 \mathrm{~cm}$ ) and video-recorded for $5 \mathrm{~min}$ to determine forelimb symmetry in exploratory rearing [23]. The video footage was analyzed by calculating the time (in seconds) that each forelimb or both forelimbs were placed on the cylinder wall. The asymmetry index is as follows: (\% ipsilateral use)-(\% contralateral use). The video analysis was performed by individuals who were blinded to these experiments.

Toluidine blue staining

Toluidine blue staining was performed on day 15 after photothrombosis [26]. Brain sections were cut into $30 \mu \mathrm{m}$ sections on a cryostat (Leica, Germany) and were collected every $90 \mu \mathrm{m}$. The slices were stained with $0.03 \%$ toluidine blue solution (Sigma, 89640 ) for 1-2 min. Then, sections were washed in distilled water, decolorized in $75 \%$ ethyl alcohol and dehydrated in $95 \%$ and $100 \%$ ethyl alcohol for 2 to $3 \mathrm{~min}$ in each step. The sections were cleared in xylene for $5 \mathrm{~min}$. Finally, the sections were mounted and observed under a light microscope (Olympus, VS120). Toluidine blue staining was traced and quantified by ImageJ.

\section{Western blot analysis}

Western blot analysis was performed as described previously [21]. Briefly, peri-infarct cortex tissues were homogenized in RIPA buffer (20 mmol/L Tris-HCl, pH 7.5, $150 \mathrm{mmol} / \mathrm{L} \mathrm{NaCl}, 1 \mathrm{mmol} / \mathrm{L}$ EDTA, $1 \%$ Triton X-100, 0.5\% sodium deoxycholate (Sigma, 30970), and 2\% Protease Inhibitor Cocktail Tablets (Roche, 04693132001)). The primary antibodies were as follows: SQSTM1 (1:1,000; Cell Signaling Technology, 5114), LC3 (1:1,000; Sigma, L7543), AMPK (1:1,000; Cell Signaling Technology, 5831), p-AMPK (T172) (1:1,000; Cell Signaling Technology, 50081), p-mTOR (S2448) (1:1000; Cell Signaling Technology, 2971), p-p70S6 (1:1000; Cell Signaling Technology, 9205), IL-1 $\beta$ (1:1000; Cell Signaling Technology, 52718), TNF-a (1:1000; Cell Signaling Technology, 11948) and GAPDH (1:3,000; Kang Chen, KC-5G4). Secondary antibodies were as follows: HRP against either rabbit or mouse lgG (1: 5,000; Cell Signaling Technology, 7071 and 7072).

Statistical analysis

All data were collected and analyzed in a blinded manner. Data are presented as the mean \pm SEM. The data were evaluated by a one-way ANOVA (analysis of variance) with Dunnett T3 post hoc tests. For behavioral testing, differences between treatment groups were analyzed using a two-way ANOVA with Tukey's multiple comparisons test for post hoc tests. $P<0.05$ was considered statistically significant.

\section{RESULTS}

Ramelteon protected against middle cerebral artery occlusion (MCAO)-induced acute ischemic brain injury

To explore the neuroprotective effect of ramelteon against acute ischemic brain injury, mice were subjected to middle cerebral artery occlusion (MCAO) (Fig. 1a). Ramelteon was administered $(0.3,1.0$ and $3.0 \mathrm{mg} / \mathrm{kg}, i . g$.) at the onset of MCAO. Brain infarct volumes and neurological deficits were determined $24 \mathrm{~h}$ after ischemia. The results showed that ramelteon reduced brain infarct volumes in a dose-dependent manner. Ramelteon at $3.0 \mathrm{mg} / \mathrm{kg}$ significantly reduced the brain infarct volumes from $52.5 \% \pm 1.7 \%$ to $32.1 \% \pm 4.6 \%$ (Fig. $1 \mathrm{~b}, \mathrm{c}$ ). In line with this finding, $3.0 \mathrm{mg} / \mathrm{kg}$ ramelteon ameliorated MCAO-induced neurological deficit (Ram $3.02 .33 \pm 0.30$ vs Vehicle $3.83 \pm 0.17, P<0.01$, Fig. 1d). These data revealed the neuroprotective role of ramelteon against MCAO-induced acute brain injury. 
a

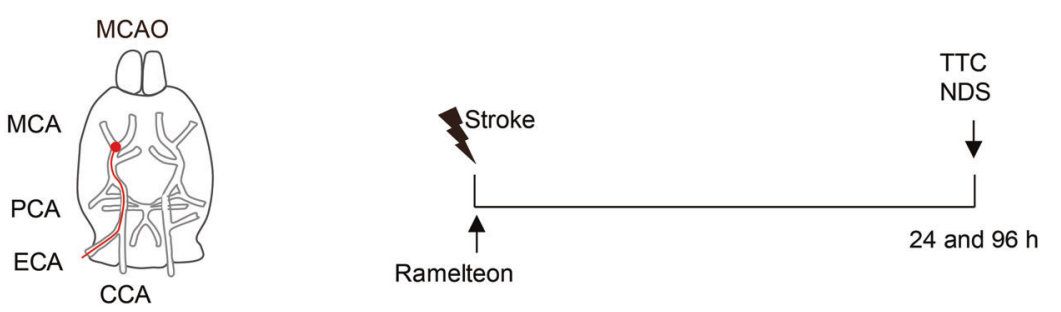

b

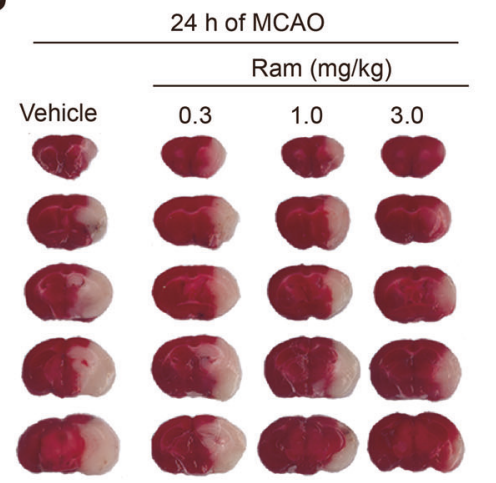

e

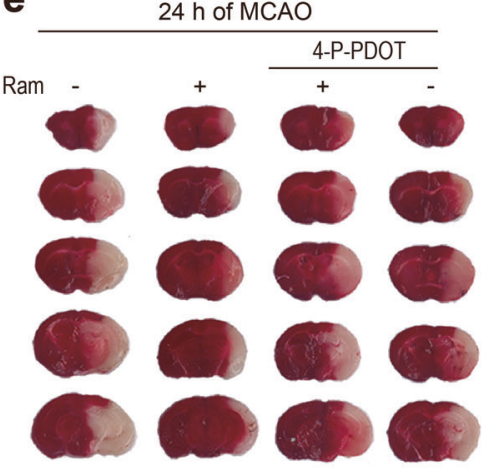

h

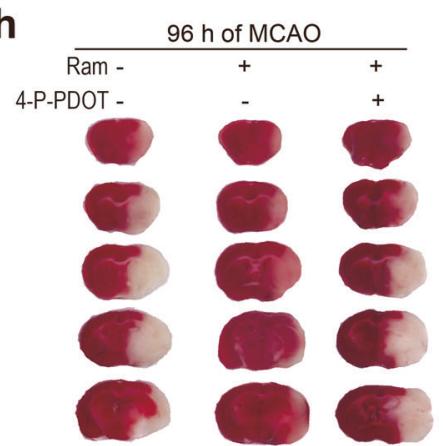

C

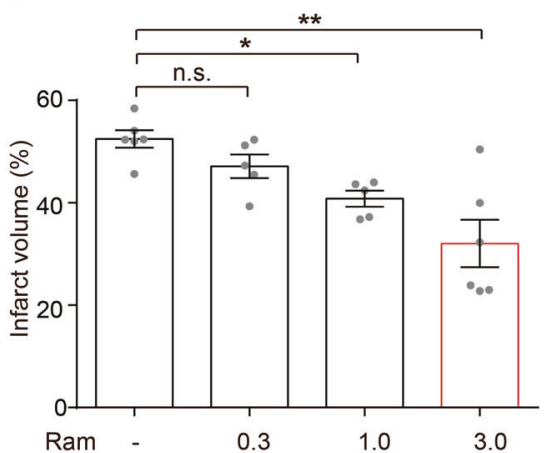

f

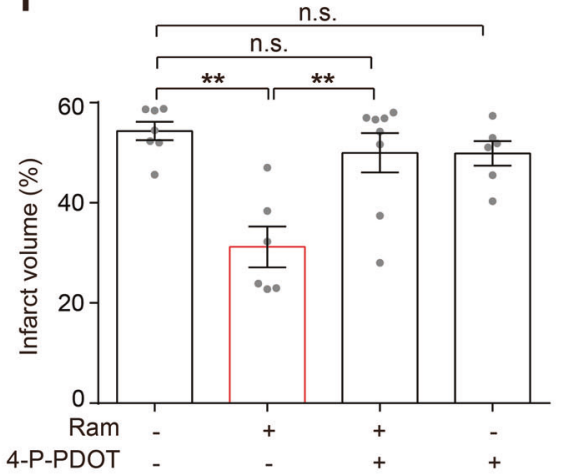

i

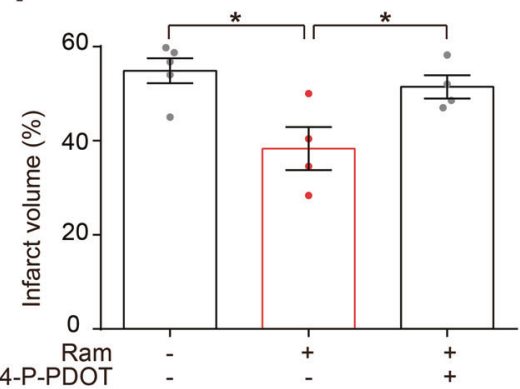

d

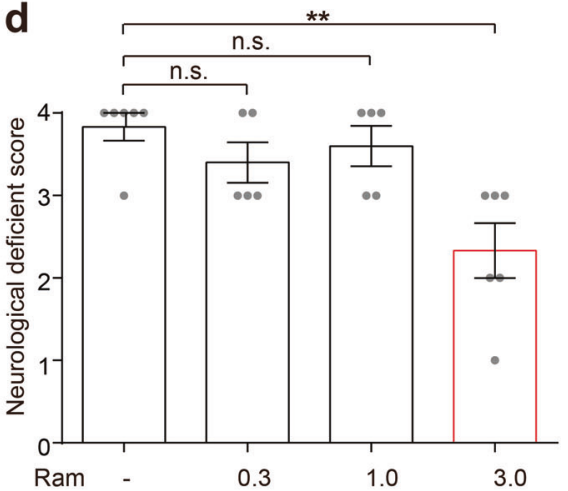

g

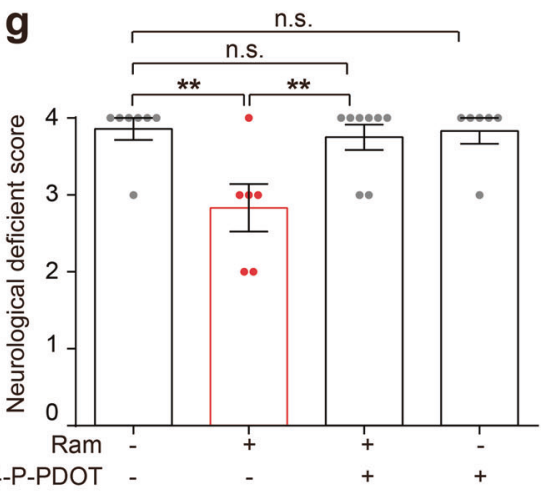

j

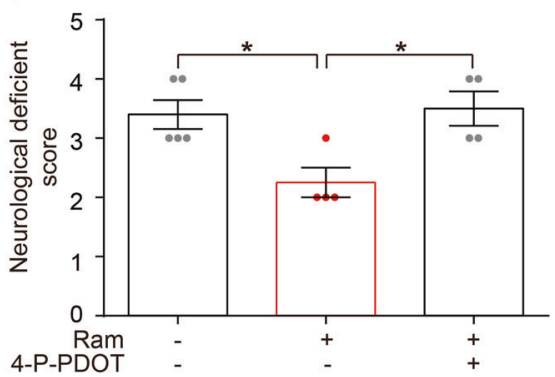

Fig. 1 Ramelteon protected against middle cerebral artery occlusion (MCAO)-induced acute ischemic brain injury. a Experimental design of acute ischemia is shown. b Ramelteon at $0.3,1.0$ or $3.0 \mathrm{mg} / \mathrm{kg}$ was administered orally at the onset of MCAO. Representative brain slices are shown after TTC staining. $\mathbf{c}$ The infarct volumes and $\mathbf{d}$ neurological deficit scores were accessed at $24 \mathrm{~h}$ after MCAO. e Mice were treated with ramelteon at $3.0 \mathrm{mg} / \mathrm{kg}, 4-P-P D O T$ at $3.0 \mathrm{mg} / \mathrm{kg}$ or cotreatment after surgery. 4-P-PDOT was injected $0.5 \mathrm{~h}$ before ramelteon administration. $n=5$ to 8 for each group. $\mathbf{f}$ The brain infarct volumes and $\mathbf{g}$ neurological deficit scores were measured as mentioned previously. $\mathbf{h}$, $\mathbf{i}$ The brain infarct volumes and $\mathbf{j}$ neurological deficit scores were measured after 96 h. $n=4$ to 5 for each group. The data are shown as the mean \pm SEM. Statistical comparisons were performed with a one-way ANOVA followed by Dunnett's multiple comparisons test. ${ }^{*} P<0.05,{ }^{* *} P<0.01 ;$ n.s. (not significant) vs the indicated group

Ramelteon is accepted as an agonist of melatonin receptors (MTs). To further clarify the involvement of MTs in neuroprotection, 4-PPDOT was employed as a specific MT antagonist. The results showed that $3.0 \mathrm{mg} / \mathrm{kg}$ 4-P-PDOT (i.p.) significantly increased the infarct volumes (4-P-PDOT + Ram $50.8 \% \pm 4.2 \%$ vs $\operatorname{Ram} 31.2 \% \pm 4.1 \%$,
$P<0.01$, Fig. 1e, $f$ ) and neurological deficit score (4-P-PDOT + Ram $3.75 \pm 0.16$ vs Ram $2.83 \pm 0.31, P<0.05$, Fig. $1 \mathrm{~g}$ ) in the ramelteon group, while 4-P-PDOT alone showed no impact on these determinations. These data indicated that 4-P-PDOT abolished ramelteon-conferred neuroprotection. We next determined the 
a

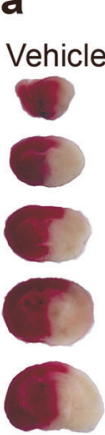

d b

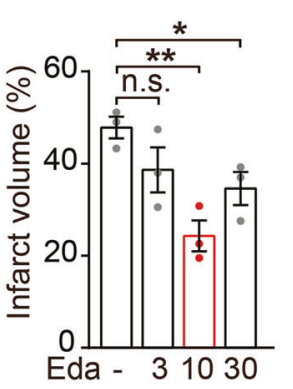

C

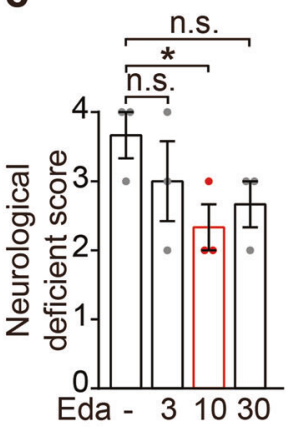

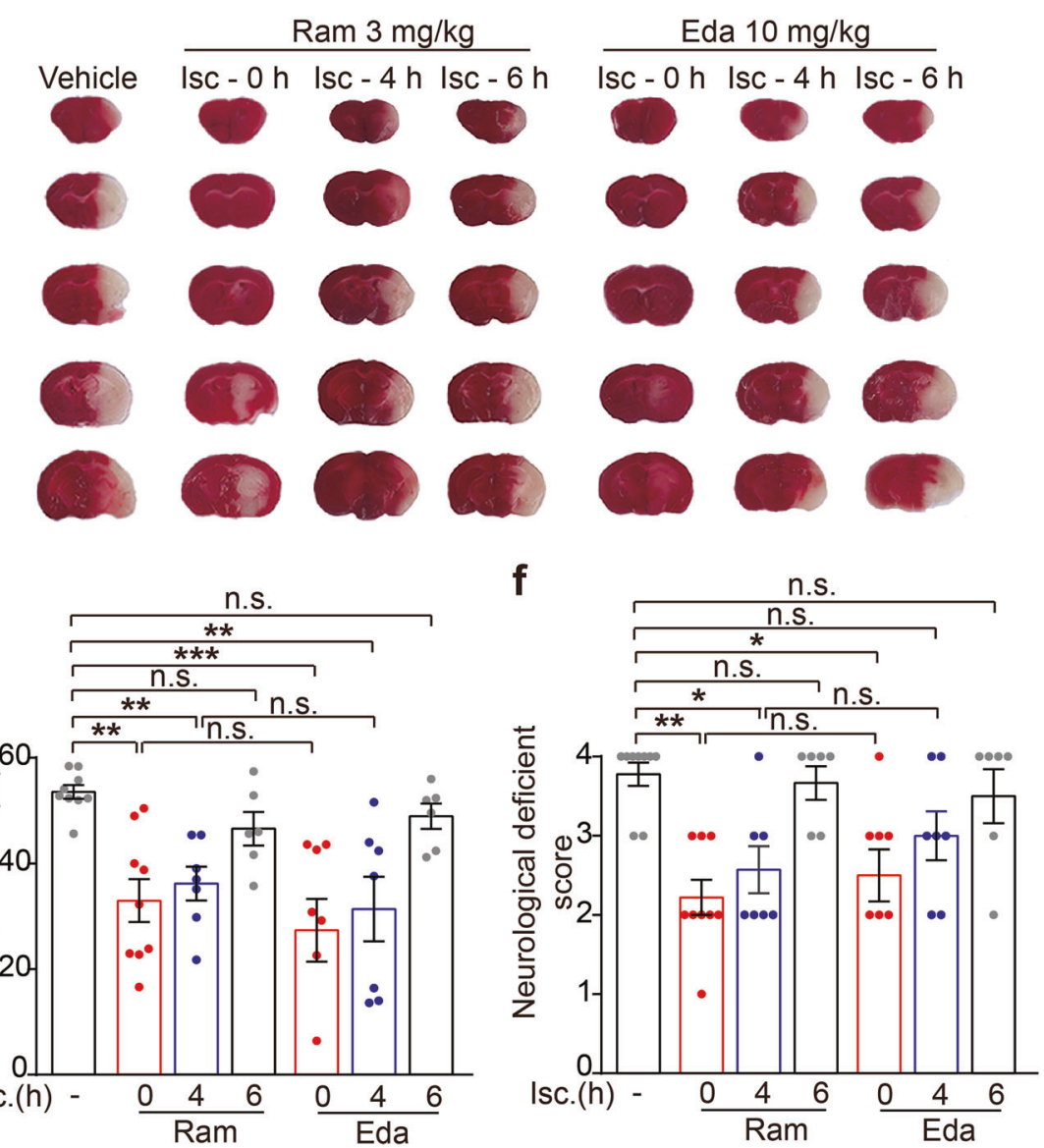

Fig. 2 Ramelteon showed comparable neuroprotective effects with edaravone in MCAO-induced acute brain injury. a Mouse brains were administered at 3,10 or $30 \mathrm{mg} / \mathrm{kg}$ edaravone and were subjected to $24 \mathrm{~h} \mathrm{pMCAO}$. Representative TTC-stained brain slices from each group are shown. $\mathbf{b}$ The infarct volumes and $\mathbf{c}$ neurological deficit scores of each group were determined $(n=3)$. $\mathbf{d}$ Ramelteon was administered at 3.0 $\mathrm{mg} / \mathrm{kg}$ at onset or at $4 \mathrm{~h}$ or $6 \mathrm{~h}$ after MCAO. Edaravone was injected intraperitoneally at $10.0 \mathrm{mg} / \mathrm{kg}$ at the same time as ramelteon treatment. Representative TTC-stained slices are shown. e Brain infarct volumes and $\mathbf{f}$ neurological deficit scores were determined $24 \mathrm{~h}$ after ischemia. Data are expressed as the mean \pm SEM, $n=6$ to 9 for each group. Statistical comparisons were performed with a one-way ANOVA followed by Dunnett's multiple comparisons test. ${ }^{*} P<0.05$, ${ }^{* *} P<0.01$, ${ }^{* *} P<0.001 ;$ n.s. (not significant) vs the indicated group

neuroprotective effect of ramelteon after 4 days of MCAO. The results showed that ramelteon significantly reduced the infarct volumes (Vehicle $54.8 \% \pm 2.6 \%$ vs Ram $38.3 \% \pm 4.6 \%, P<0.05$, Fig. $1 \mathrm{~h}, \mathrm{i}$ ) and neurological deficit scores (Vehicle $3.4 \pm 0.24$ vs Ram $2.3 \pm 0.25, P<0.05$, Fig. 1j). We found that ramelteon still had a neuroprotective role in prolonged ischemic brain injury.

Effective time window of ramelteon in reducing MCAO-induced brain injury

To explore the time window during which ramelteon can reduce acute ischemic brain injury. Ramelteon at $3.0 \mathrm{mg} / \mathrm{kg}$ was administered at the onset $(0 \mathrm{~h})$ or at $4 \mathrm{~h}$ and $6 \mathrm{~h}$ after ischemia onset. Ramelteon significantly reduced brain infarct volumes as late as $4 \mathrm{~h}$ after ischemia (Ram $4 \mathrm{~h} 36.2 \% \pm 3.2 \%$ vs Vehicle $54.3 \% \pm 1.9 \%, P<0.05$, Fig. 2 d, e). Consistent with this observation, ramelteon significantly reversed neurological deficit when administered as late as $4 \mathrm{~h}$ after MCAO (Ram $4 \mathrm{~h} 2.6 \pm 0.29$ vs Vehicle $3.9 \pm 0.18, P<0.01$, Fig. $2 f)$. We further prolonged the administration time to $6 \mathrm{~h}$ after MCAO. The results showed that $3.0 \mathrm{mg} / \mathrm{kg}$ ramelteon failed to rescue infarct volumes (Ram $6 \mathrm{~h} 48.2 \% \pm 4.5 \%$ vs Vehicle $54.3 \% \pm 1.9 \%, P=0.16$, Fig. 2e) and neurological deficit (Ram $6 \mathrm{~h} 3.7 \pm 0.21$ vs Vehicle $3.9 \pm 0.18, P=0.42$, Fig. 2f). These data indicated that the effective time window of ramelteon can be as long as $4 \mathrm{~h}$ in MCAO mice. 
Comparison of the neuroprotective effect of ramelteon and edaravone in MCAO-induced acute brain injury

We next compared the neuroprotection of ramelteon with edaravone, a well-accepted therapy for stroke patients, in the MCAO model. The neuroprotective effect of edaravone against ischemia was reported as previously described [27, 28]. To optimize the dose of edaravone in our model, mice were intraperitoneally administered edaravone at 3,10 , and $30 \mathrm{mg} / \mathrm{kg}$ at the onset of MCAO. Edaravone at $10.0 \mathrm{mg} / \mathrm{kg}$ showed the maximal efficacy in reducing infarct volumes (Eda $1024.31 \% \pm$ $3.3 \%$ vs Vehicle $47.82 \% \pm 2.3 \%, P<0.01$, Fig. $2 a$, b) and NDS (Eda $102.33 \pm 0.33$ vs Vehicle $3.6 \pm 0.34, P<0.05$, Fig. 2 c). The results showed that $10 \mathrm{mg} / \mathrm{kg}$ edaravone significantly reduced brain infarct volumes and neurological deficits; nevertheless, edaravone showed no significant advantages in ameliorating MCAO-induced brain injury compared with ramelteon. We further determined the neuroprotective effect of edaravone and ramelteon by prolonging the administration time to 4 and $6 \mathrm{~h}$. Edaravone showed no advantage over ramelteon in reducing MCAO-induced injury by delayed administration, as reflected by brain infarct volumes and NDS (Fig. 2e, f). These data suggested that ramelteon provided a similar effective time window as edaravone for stroke therapy.

Ramelteon protected against photothrombosis (PT)-induced chronic ischemic brain injury

To determine whether ramelteon may also attenuate chronic ischemic brain injury, mice were subjected to photothrombosis. Ramelteon $(3.0 \mathrm{mg} / \mathrm{kg})$ was administered daily after photothrombosis for 7 days, and the volumes of lesioned brains were evaluated 15 days after ischemia (Fig. 3a). The results showed that ramelteon significantly reduced the brain lesion from $0.76 \pm 0.14$ $\mathrm{mm}^{3}$ to $0.38 \pm 0.13 \mathrm{~mm}^{3}(P<0.05)$. Additionally, the protective effect of ramelteon was counteracted by 4-P-PDOT, suggesting the involvement of MTs in the neuroprotection of ramelteon (Fig. 3b, c). To determine the impairment of motor function, we measured the number of foot faults in the grid-walking task and forelimb symmetry in the cylinder task every other day. We found that ramelteon significantly reduced the number of foot faults and forelimb symmetry during the 15 days after PT onset. Likewise, the functional recovery with ramelteon treatment was abolished by 4-P-PDOT treatment (Fig. 3d, e). Taken together, these data clearly indicated that ramelteon protected against chronic cerebral ischemia and promoted functional recovery in an MT-related manner.

The AMPK/mTOR signaling pathway and autophagy may be involved in ramelteon-conferred neuroprotection

Ischemic brain injury is intimately related to autophagy cell death. It has been shown that MT activation regulates the AMPK/mTOR signaling pathway [29]. As expected, ramelteon significantly decreased the cerebral-ischemia-induced phosphorylation of AMPK (T172) and increased the phosphorylation of mTOR (S2448) and p70S6 kinase, which were abolished by 4-P-PDOT (Fig. 4a, b). AMPK/mTOR signaling has been accepted to activate autophagy in the context of cerebral ischemia [30], and there are studies indicating that MT agonists regulate autophagy in ischemic brains [31, 32]. We thus hypothesized that ramelteon conferred its neuroprotection by modulating autophagy. To this end, autophagy-related proteins SQSTM1 and LC3 were determined. Autophagy activation was detected in the brain tissue subjected to MCAO, as reflected by reduced SQSTM1 and accumulated LC3-II. Ramelteon treatment significantly prevented these alterations, indicating that ramelteon inhibited autophagy activation in acute ischemic brain tissue. These effects were reversed by 4-P-PDOT, further suggesting the involvement of MTs in autophagy regulation (Fig. 4c, d). These data support the notion that ramelteon may regulate autophagy activation by modulating the $A M P K / m T O R$ signaling pathway. To further determine the a
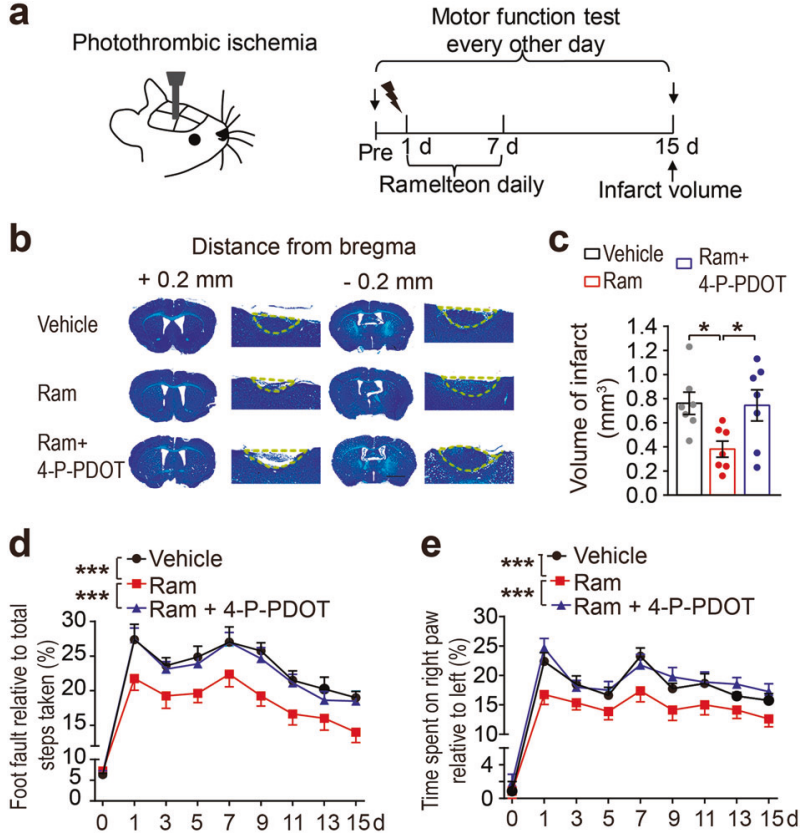

Fig. 3 Ramelteon protected against photothrombosis (PT)-induced chronic ischemic brain injury. a Schematic of the experimental protocol. b Mice were treated with ramelteon at $3.0 \mathrm{mg} / \mathrm{kg}$ daily for 7 days after photothrombosis. 4-P-PDOT was injected $0.5 \mathrm{~h}$ before ramelteon treatment. Fifteen days after PT onset, brain sections were stained with toluidine blue. Representative images are shown, and the dashed lines outline the brain lesion in the cortex. Data are expressed as the mean \pm SEM, $n=7$ for each group. Scale bar: $2 \mathrm{~mm}$. c The infarct volumes were quantified and expressed as $\mathrm{mm}^{3}$. Statistical comparisons were performed with a one-way ANOVA followed by Dunnett's multiple comparisons test. $\mathbf{d}$ Motor functions were accessed by foot fault test and e cylinder task. Behavioral tests were performed every two days after stroke. $n=8-10$ for each group. Statistical comparisons were performed with a two-way ANOVA followed by Tukey's multiple comparisons test. ${ }^{*} P<0.05$, ${ }^{* * *} P<0.001$ vs the indicated group

involvement of autophagy inhibition in the neuroprotection of ramelteon, rapamycin was administered as an autophagy activator. The neuroprotection of ramelteon was abolished by rapamycin, as demonstrated by increased infarct volumes (Ram $33.41 \% \pm 4.3 \%$ vs Rapa $52.1 \% \pm 0.9 \%, P<0.01$ ) and NDS (Ram $2.9 \pm 0.23$ Rapa vs $4.0 \pm 0.26, P<0.05$, Fig. 4 e, f)

We next asked whether the AMPK/mTOR signaling pathway and autophagy activation can also be regulated by ramelteon in chronic ischemia. We found that p-AMPK (T172) was upregulated and p-mTOR (S2448) and p-p70S6 were downregulated at 15 days after ischemia. Ramelteon prevented the phosphorylation of AMPK (T172) and the decrease in p-mTOR (S2448) and p-p70S6, while these effects were abrogated by 4-P-PDOT (Fig. 5a, b). Autophagy was still activated, as reflected by increased LC3-II and decreased SQSTM1. Ramelteon counteracted autophagy activation, which was further abolished by 4-P-PDOT (Fig. 5c, d). Overall, these data supported the involvement of the AMPK/mTOR signaling pathway and autophagy in ramelteon-conferred neuroprotection in either acute or chronic ischemic brain injury. mTOR signaling was reported to regulate neuroinflammation [33, 34], which is involved in the pathological process of cerebral ischemia [35]. Melatonin attenuated ischemic brain injury through its antiinflammatory properties [36]. We also examined whether ramelteon protected cerebral ischemia through anti-inflammation. After ischemia, the increased levels of IL-1 $\beta$ and TNF- $a$ were suppressed by ramelteon treatment (Fig. S1). The results indicated that ramelteon might attenuate inflammation in cerebral ischemia. 
a
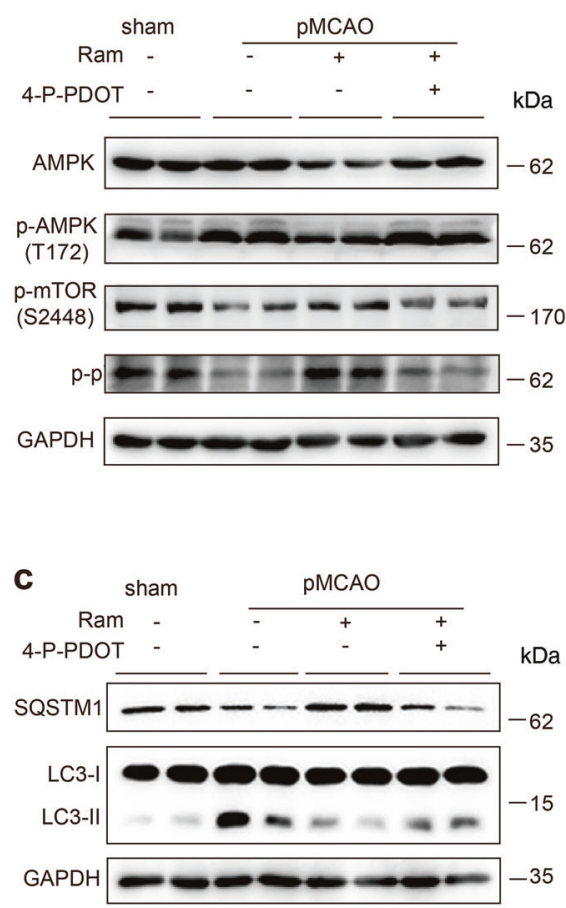

e

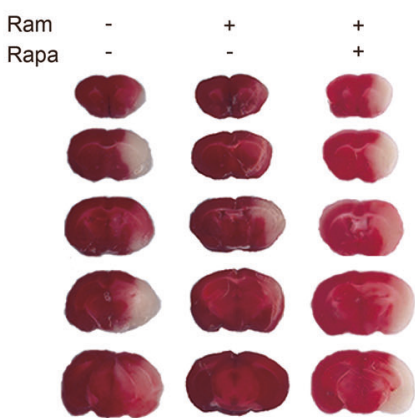

b
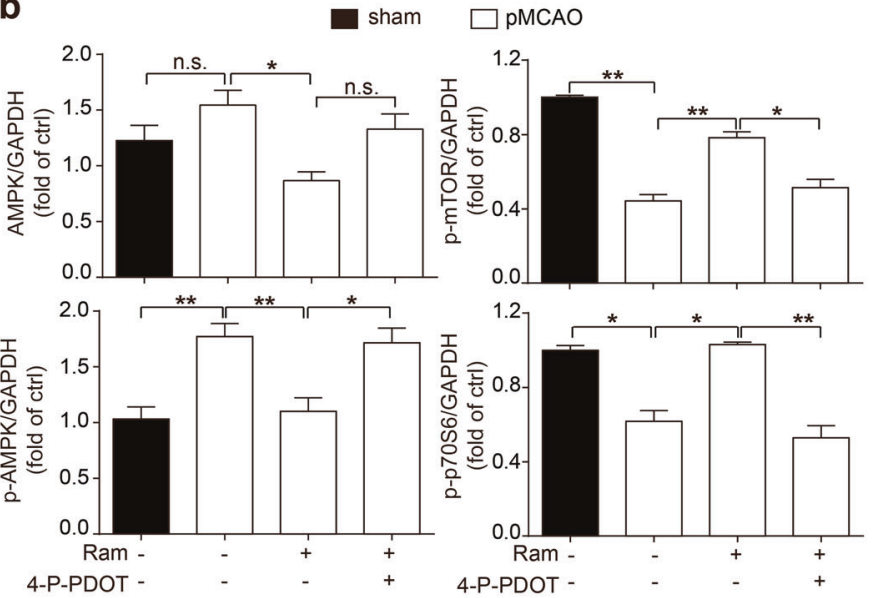

d
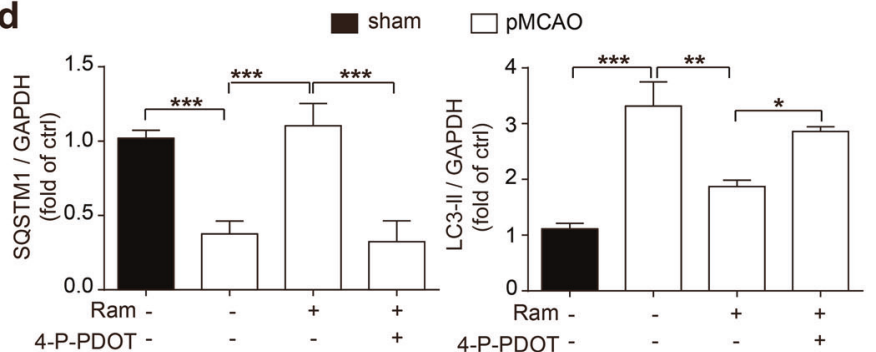

f
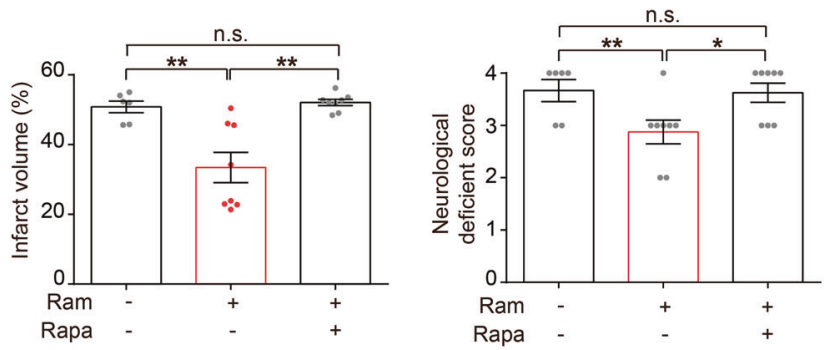

Fig. 4 Ramelteon activated the AMPK/mTOR signaling pathway and inhibited autophagy in acute ischemic brain injury. a Mice were administered ramelteon at $3.0 \mathrm{mg} / \mathrm{kg}$ or were cotreated with 4-P-PDOT at $3.0 \mathrm{mg} / \mathrm{kg}$ after MCAO. The expression of AMPK, p-AMPK (T172), p-mTOR (S2448), p-p70S6 and GAPDH in the ischemic penumbra was measured by Western blot analysis $24 \mathrm{~h}$ after MCAO. $n=4$ for each group. b Semiquantitative analyses of AMPK, p-AMPK (T172), p-mTOR (S2448), and p-p70S6 are shown. c The expression of SQSTM1 and LC3B was determined by Western blot analysis. $\mathbf{d}$ Semiquantitative analyses of SQSTM1 and LC3-II are shown. e Mice were treated with ramelteon at $3.0 \mathrm{mg} / \mathrm{kg}$ or were cotreated with rapamycin at $10.0 \mu \mathrm{mol}$ at the onset of ischemia. Rapamycin was dissolved in saline and intracerebroventricularly (i.c.v.) injected. $\mathbf{f}$ Infarct volumes and NDS were accessed as previously described. $n=8-10$. The data are expressed as the mean \pm SEM. Statistical comparisons were performed with a one-way ANOVA followed by Dunnett's multiple comparisons test. ${ }^{*} P<0.05,{ }^{* *} P<0.01,{ }^{* *} P<0.001 ;$ n.s. (not significant) $v s$ the indicated group

The molecular mechanism of ramelteon in anti-inflammation needs to be investigated in future studies. Our results showed that ramelteon protected against both acute and chronic ischemic injury. Ramelteon may activate the AMPK/mTOR signaling pathway and inhibit ischemia-induced autophagy in a melatonin receptor-related manner (Fig. 6).

\section{DISCUSSION}

Ramelteon is a hypnotic agent that activates melatonin receptors. Some emerging studies have implied other clinical applications beside sedation [19]; however, the neuroprotective effect of ramelteon against ischemic injury has never been tested. In the present study, we reported for the first time that ramelteon attenuated brain injury caused by either acute or chronic brain ischemia. MCAO was the model used to mimic acute brain ischemia. Ramelteon showed significant neuroprotection in reducing MCAO-induced infarct volume and NDS in a dosedependent manner. We also found that ramelteon still protected against ischemia in the brain 4 days after MCAO onset (Fig. 1). Likewise, ramelteon attenuated brain lesions induced by chronic ischemia, and neuroprotection was observed at least 15 days after ischemia onset (Fig. 3). We cannot exclude the ineffectiveness of ramelteon in the time range longer than 15 days in our study. To evaluate the neuroprotection of ramelteon more comprehensively, we compared its efficacy with that of edaravone (Fig. 2), which is an approved drug for stroke therapy [37, 38]. We found that ramelteon showed neuroprotective effects that were comparable with those of edaravone against acute ischemic injury. This observation suggested that ramelteon might have equivalent efficacy with edaravone for stroke therapy. Considering the safety of ramelteon in clinical application, our data indicated 
a

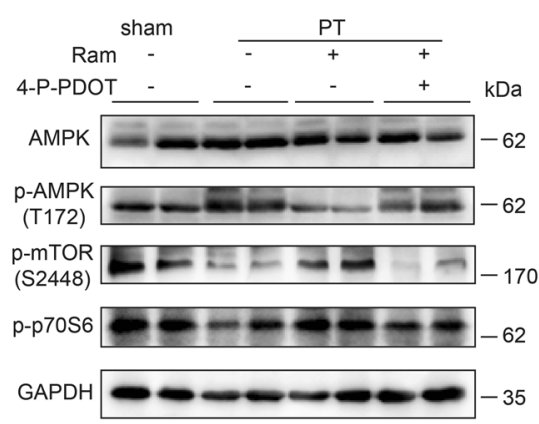

C

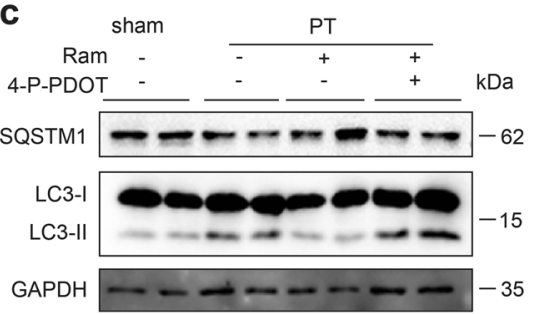

b
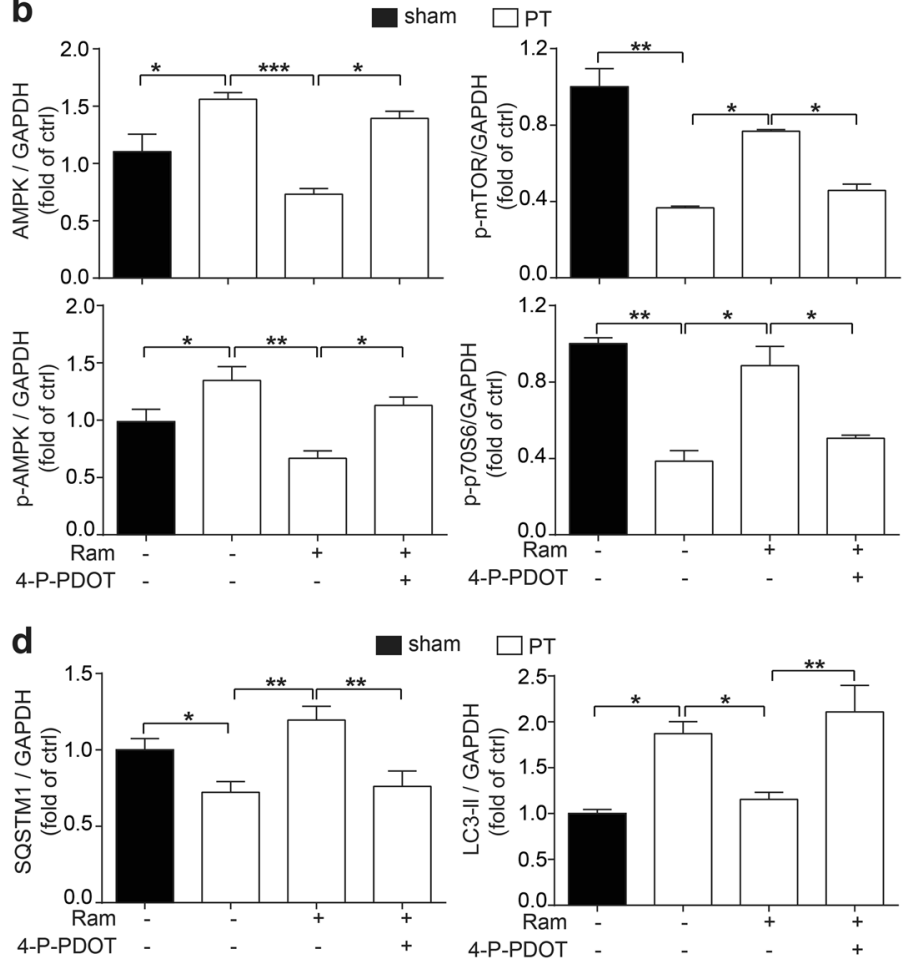
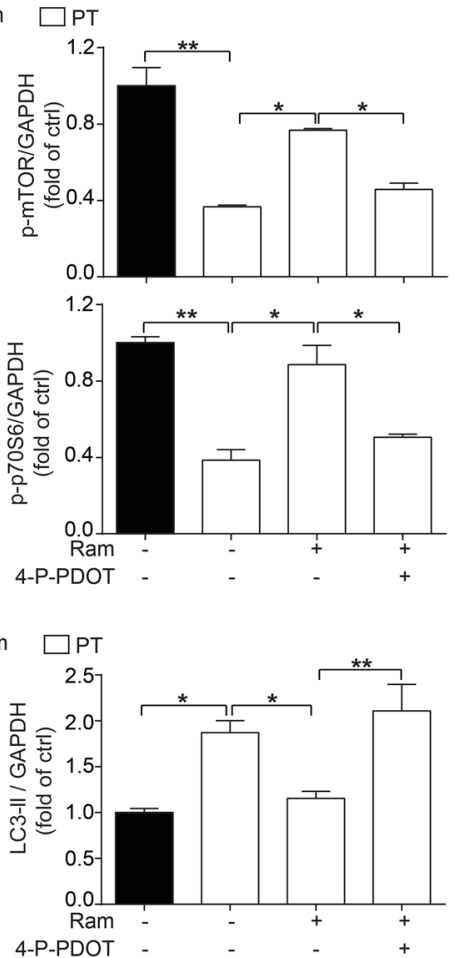

Fig. 5 Ramelteon activated the AMPK/mTOR signaling pathway and suppressed autophagy in chronic ischemic brain injury. a Mice were subjected to photothrombosis, and 15 days later, the expression of AMPK, p-AMPK (T172), p-mTOR (S2448), p-p70S6 and GAPDH in the periinfarct cortex was examined by Western blot analysis. b Semiquantitative analyses are shown. $n=4$ for each group. $\mathbf{c}$ The expression of SQSTM1 and LC3B was determined by Western blot analysis. d Semiquantitative analyses of SQSTM1 and LC3-II are shown. The data are expressed as the mean \pm SEM. Statistical comparisons were performed with a one-way ANOVA followed by Dunnett's multiple comparisons test. ${ }^{*} P<0.05,{ }^{* *} P<0.01,{ }^{* *} P<0.001$ vs the indicated group

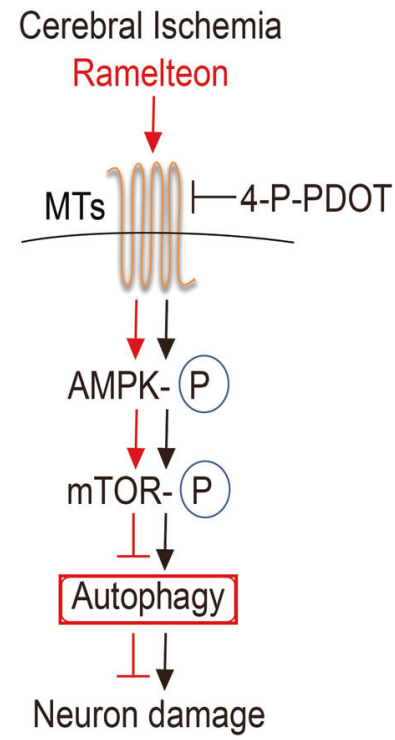

Fig. 6 Diagram of the neuroprotective effects of ramelteon on cerebral ischemic injury. Ramelteon inhibited the cerebral-ischemiainduced activation of autophagy through the AMPK/mTOR signaling pathway. These effects were blocked by the melatonin receptor inhibitor 4-P-PDOT

that ramelteon can be a novel potential therapeutic drug for cerebral ischemia. We optimized the dose of ramelteon and found that $3.0 \mathrm{mg} / \mathrm{kg}$ showed the maximal efficacy (data not shown). We found that $3.0 \mathrm{mg} / \mathrm{kg}$ of ramelteon was able to attenuate both acute and chronic brain ischemia. By converting body surface area, this dose is $\sim 19 \mathrm{mg}$ in humans ( $60 \mathrm{~kg}$ body weight). This dose is equivalent to that for insomnia therapy, which ranges from 8 to $32 \mathrm{mg} /$ day $[18,39]$. Approximately $30 \%$ of stroke patients suffered from sleep problems, including delirium and insomnia $[40,41]$. Given the hypnotic purpose of ramelteon at this dose, ramelteon may have advantages in treating patients with destabilized circadian rhythms after stroke $[42,43]$. In addition, a narrow therapeutic window is one of the most challenging issues in stroke therapy [2, 4]. Ramelteon showed an effective therapeutic time window as long as $4 \mathrm{~h}$ in this mouse MCAO model. This effective therapeutic window was equal to that of edaravone (Fig. 2) [28], which further supported the potential clinical application of ramelteon in stroke therapy.

Lines of evidence documented the neuroprotective effect of melatonin against cerebral ischemia [32]. MTs are the primary targets of melatonin; however, it is controversial whether MTs are required for melatonin to rescue ischemic brains [44, 45]. Here, we used 4-P-PDOT to block MTs $[25,46]$. Although 4-P-PDOT has been frequently used as a selective MT2 antagonist, its selectivity between MT1 and MT2 was poor at the current dose [47]. We found that the neuroprotection of ramelteon was almost totally abolished by 4-P-PDOT both in acute and chronic ischemia (Figs. 1, $3)$, which supported the requirement of MTs for the neuroprotection of ramelteon. Some recent studies indicated that MT1 and MT2 may conduct diverse signaling in ischemic brains [14, 48]. Due to the lack of selective MT antagonists, it remains unclear how MTs may contribute to the neuroprotection of ramelteon. This issue could be addressed by employing MT1 and MT2 gene knockout mice in future studies. Additionally, previous studies indicated the requirement of melatonin pretreatment before an ischemic episode [49], and a delay of administration abolished the neuroprotection of melatonin against acute ischemia [50]. It took 
hours for melatonin to reach its therapeutic level in a nonrodent stroke animal model [51]. Given the sudden occurrence of ischemic stroke, melatonin may still have disadvantages as a rescuing agent for ischemic brain injury. As a comparison, we found that ramelteon was effective even $4 \mathrm{~h}$ after MCAO onset. This result suggested that ramelteon could be a therapeutic drug, while the prophylactic effect of melatonin should be emphasized for acute ischemia intervention. The discrepancy may be attributed to the advantages in pharmacokinetics and to the fact that ramelteon has a higher affinity than melatonin to MTs $[47,52,53]$.

Autophagy activation has been accepted as a critical mechanism underlying ischemic neuronal death, particularly in ischemic brains without reperfusion [54, 55]. Additionally, AMPK/mTOR signaling was accepted to activate autophagy during cerebral ischemia [56]. Our data confirmed autophagy activation in ischemic brains by showing upregulated LC3-II and decreased SQSTM1 and AMPK/mTOR inhibition (Fig. 4a, c). Ramelteon suppressed the autophagy activation induced by ischemia. In addition, rapamycin, an autophagy activator, abolished the ramelteon-conferred neuroprotection (Fig. 4e, f). It remains a controversial issue whether rapamycin is neuroprotective in cerebral ischemia, yet a variety of studies have confirmed its role as an autophagy activator [57-59]. Nevertheless, due to the lack of specific approaches to activating autophagy, we cannot exclude other mechanisms underlying the neuroprotection of ramelteon in addition to autophagy inhibition. Nevertheless, the present study indicated that autophagy may be involved in ramelteon-conferred protection. Likewise, melatonin has been demonstrated to regulate autophagy activation in ischemic rats [60]. However, it was unclear whether MTs are required for autophagy regulation by melatonin. It seems that MTs regulate neuronal autophagy via AMPK/mTOR signaling, which is activated in ischemic conditions [61]. Here, we found that MT blockage by 4-P-PDOT largely prevented AMPK/mTOR activation and autophagy induction (Figs. 4 and 5). Therefore, the present study supported the involvement of MTs in autophagy regulation. In addition, melatonin was reported to counteract ischemia-induced autophagy $[7,62]$. In these studies, pretreatment with melatonin was required for its neuroprotection, and the benefits of melatonin against chronic stroke have not been determined. We found that ramelteon could be neuroprotective as late as $4 \mathrm{~h}$ after acute ischemia and be beneficial in chronic cerebral ischemia. These data implied the advantages of ramelteon over melatonin as a therapeutic drug for stroke.

Taken together, we provide the first evidence indicating that ramelteon can be a promising therapeutic drug for stroke therapy. The neuroprotective effect of ramelteon may be attributed to its agonism on MTs and its inhibition of autophagy in ischemic brains.

\section{ACKNOWLEDGEMENTS}

This work was funded by the National Natural Science Foundation of China (81822044, 81773703, and 81872844), the Natural Science Foundation of Zhejiang Province (LY19H310007), and the Fundamental Research Funds for the Central Universities (2019XZZX004-17).

\section{AUTHOR CONTRIBUTIONS}

ZC and XNZ designed the project; XLW and SSL performed most of the surgical experiments, imaging and immunoblotting; MRL, JZC, YRZ and WDT contributed to analyzing the data in a blinded manner; $A A, M C$ and $\amalg$ helped to perform the MCAO model and to raise mice; WWH and JYW contributed to organizing the results and discussion; and ZC and XNZ wrote the manuscript with input from all other authors.

\section{ADDITIONAL INFORMATION}

The online version of this article (https://doi.org/10.1038/s41401-020-0361-2) contains supplementary material, which is available to authorized users.

Competing interests: The authors declare no competing interests.

\section{REFERENCES}

1. Yenari MA, Han HS. Neuroprotective mechanisms of hypothermia in brain ischaemia. Nat Rev Neurosci. 2012;13:267-78.

2. Zerna C, Thomalla G, Campbell BCV, Rha JH, Hill MD. Current practice and future directions in the diagnosis and acute treatment of ischaemic stroke. Lancet. 2018:392:1247-56.

3. Liang J, Qi Z, Liu W, Wang P, Shi W, Dong W, et al. Normobaric hyperoxia slows bloodbrain barrier damage and expands the therapeutic time window for tissue-type plasminogen activator treatment in cerebral ischemia. Stroke. 2015;46:1344-51.

4. Minnerup J, Wersching H, Ringelstein EB, Schilling M, Schabitz WR, Wellmann J, et al. Impact of the extended thrombolysis time window on the proportion of recombinant tissue-type plasminogen activator-treated stroke patients and on door-to-needle time. Stroke. 2011;42:2838-43.

5. Catanese L, Tarsia J, Fisher M. Acute ischemic stroke therapy overview. Circ Res. 2017;120:541-58

6. Jackman K, ladecola C. Neurovascular regulation in the ischemic brain. Antioxid Redox Signal. 2015;22:149-60.

7. Feng D, Wang B, Wang L, Abraham N, Tao K, Huang L, et al. Pre-ischemia melatonin treatment alleviated acute neuronal injury after ischemic stroke by inhibiting endoplasmic reticulum stress-dependent autophagy via PERK and IRE1 signalings. J Pineal Res. 2017;62:e12395.

8. Guerrero JM, Reiter RJ, Ortiz GG, Pablos MI, Sewerynek E, Chuang Jl. Melatonin prevents increases in neural nitric oxide and cyclic GMP production after transient brain ischemia and reperfusion in the Mongolian gerbil (Meriones unguiculatus). J Pineal Res. 1997;23:24-31.

9. Sinha K, Degaonkar MN, Jagannathan NR, Gupta YK. Effect of melatonin on ischemia reperfusion injury induced by middle cerebral artery occlusion in rats. Eur J Pharmacol. 2001;428:185-92.

10. Li X, Tao RR, Hong LJ, Cheng J, Jiang Q, Lu YM, et al. Visualizing peroxynitrite fluxes in endothelial cells reveals the dynamic progression of brain vascular injury. J Am Chem Soc. 2015;137:12296-303.

11. Tao RR, Huang JY, Shao XJ, Ye WF, Tian Y, Liao MH, et al. Ischemic injury promotes Keap1 nitration and disturbance of antioxidative responses in endothelial cells: a potential vasoprotective effect of melatonin. J Pineal Res. 2013;54:271-81.

12. Zhao Z, Lu C, Li T, Wang W, Ye W, Zeng R, et al. The protective effect of melatonin on brain ischemia and reperfusion in rats and humans: In vivo assessment and a randomized controlled trial. J Pineal Res. 2018;65:e12521.

13. Lee CH, Yoo KY, Choi JH, Park OK, Hwang IK, Kwon YG, et al. Melatonin's protective action against ischemic neuronal damage is associated with up-regulation of the MT2 melatonin receptor. J Neurosci Res. 2010;88:2630-40.

14. Tang Y, Cai B, Yuan F, He X, Lin X, Wang J, et al. Melatonin pretreatment improves the survival and function of transplanted mesenchymal stem cells after focal cerebral ischemia. Cell Transpl. 2014;23:1279-91.

15. Buendia I, Gomez-Rangel V, Gonzalez-Lafuente L, Parada E, Leon R, Gameiro I, et al. Neuroprotective mechanism of the novel melatonin derivative Neu-P11 in brain ischemia related models. Neuropharmacology. 2015;99:187-95.

16. O'Neill B, Gardani M, Findlay G, Whyte T, Cullen T. Challenging behaviour and sleep cycle disorder following brain injury: a preliminary response to agomelatine treatment. Brain Inj. 2014;28:378-81.

17. Laustsen G, Andersen M. Ramelteon (rozerem) a novel approach for insomnia treatment. Nurse Pr. 2006;31:52-5.

18. Edmonds C, Swanoski M. A review of suvorexant, doxepin, ramelteon, and tasimelteon for the treatment of insomnia in geriatric patients. Consult Pharm. 2017;32:156-60.

19. Stroethoff M, Christoph I, Behmenburg F, Raupach A, Bunte S, Senpolat $S$, et al Melatonin receptor agonist ramelteon reduces ischemia-reperfusion injury through activation of mitochondrial potassium channels. J Cardiovasc Pharmacol. 2018;72:106-11.

20. Zhang X, Yan H, Yuan Y, Gao J, Shen Z, Cheng Y, et al. Cerebral ischemiareperfusion-induced autophagy protects against neuronal injury by mitochondrial clearance. Autophagy. 2013;9:1321-33.

21. Shen Z, Zheng Y, Wu J, Chen Y, Wu X, Zhou Y, et al. PARK2-dependent mitophagy induced by acidic postconditioning protects against focal cerebral ischemia and extends the reperfusion window. Autophagy. 2017;13:473-85.

22. Yuan $Y$, Zheng $Y$, Zhang $X$, Chen $Y, W u ~ X, W u ~ J$, et al. BNIP3L/NIX-mediated mitophagy protects against ischemic brain injury independent of PARK2. Autophagy. 2017;13:1754-66. 
23. Lin $Y H$, Dong J, Tang $Y$, Ni HY, Zhang $Y$, Su $P$, et al. Opening a new time window for treatment of stroke by targeting HDAC2. J Neurosci. 2017;37:6712-28.

24. Clarkson AN, Overman JJ, Zhong S, Mueller R, Lynch G, Carmichael ST. AMPA receptor-induced local brain-derived neurotrophic factor signaling mediates motor recovery after stroke. J Neurosci. 2011;31:3766-75.

25. Shin EJ, Chung YH, Le HL, Jeong JH, Dang DK, Nam Y, et al. Melatonin attenuates memory impairment induced by Klotho gene deficiency via interactive signaling between MT2 receptor, ERK, and Nrf2-related antioxidant potential. Int J Neuropsychopharmacol. 2014; 18:105.

26. Zhu Y, Liu F, Zou X, Torbey M. Comparison of unbiased estimation of neuronal number in the rat hippocampus with different staining methods. J Neurosci Methods. 2015;254:73-9.

27. Zhang D, Xiao Y, Lv P, Teng Z, Dong Y, Qi Q, et al. Edaravone attenuates oxidative stress induced by chronic cerebral hypoperfusion injury: role of ERK/Nrf2/HO1 signaling pathway. Neurol Res. 2018;40:1-10.

28. Yamaguchi T, Awano H, Matsuda H, Tanahashi N, Investigators P. Edaravone with and without $.6 \mathrm{Mg} / \mathrm{Kg}$ Alteplase within $4.5 \mathrm{~h}$ after Ischemic Stroke: A Prospective Cohort Study (PROTECT4.5). J Stroke Cerebrovasc Dis. 2017;26:756-65.

29. Wang Y, Guo W, Xu H, Tang K, Zan L, Yang W. Melatonin suppresses milk fat synthesis by inhibiting the mTOR signaling pathway via the MT1 receptor in bovine mammary epithelial cells. J Pineal Res. 2019;67:e12593.

30. Guo Z, Cao G, Yang H, Zhou H, Li L, Cao Z, et al. A combination of four active compounds alleviates cerebral ischemia-reperfusion injury in correlation with inhibition of autophagy and modulation of AMPK/mTOR and JNK pathways. J Neurosci Res. 2014;92:1295-306.

31. Zhou H, Wang J, Jiang J, Stavrovskaya IG, Li M, Li W, et al. N-acetyl-serotonin offers neuroprotection through inhibiting mitochondrial death pathways and autophagic activation in experimental models of ischemic injury. J Neurosci. 2014;34:2967-78.

32. Luo C, Yang Q, Liu Y, Zhou S, Jiang J, Reiter RJ, et al. The multiple protective roles and molecular mechanisms of melatonin and its precursor $\mathrm{N}$-acetylserotonin in targeting brain injury and liver damage and in maintaining bone health. Free Radic Biol Med. 2019:130:215-33.

33. Russo E, Andreozzi F, luliano R, Dattilo V, Procopio T, Fiume G, et al. Early molecular and behavioral response to lipopolysaccharide in the WAG/Rij rat model of absence epilepsy and depressive-like behavior, involves interplay between AMPK, AKT/mTOR pathways and neuroinflammatory cytokine release. Brain Behav Immun. 2014;42:157-68.

34. Xie L, Sun F, Wang J, Mao X, Xie L, Yang SH, et al. mTOR signaling inhibition modulates macrophage/microglia-mediated neuroinflammation and secondary injury via regulatory T cells after focal ischemia. J Immunol. 2014;192:6009-19.

35. Kawabori M, Yenari MA. Inflammatory responses in brain ischemia. Curr Med Chem. 2015;22:1258-77.

36. Wang X, Figueroa BE, Stavrovskaya IG, Zhang $Y$, Sirianni AC, Zhu S, et al. Methazolamide and melatonin inhibit mitochondrial cytochrome $C$ release and are neuroprotective in experimental models of ischemic injury. Stroke. 2009;40:1877-85.

37. Enomoto $\mathrm{M}$, Endo A, Yatsushige $\mathrm{H}$, Fushimi $\mathrm{K}$, Otomo $\mathrm{Y}$. Clinical effects of early edaravone use in acute ischemic stroke patients treated by endovascular reperfusion therapy. Stroke. 2019;50:652-8.

38. Wu S, Sena E, Egan K, Macleod M, Mead G. Edaravone improves functional and structural outcomes in animal models of focal cerebral ischemia: a systematic review. Int J Stroke. 2014;9:101-6.

39. Erman M, Seiden D, Zammit G, Sainati S, Zhang J. An efficacy, safety, and doseresponse study of Ramelteon in patients with chronic primary insomnia. Sleep Med. 2006;7:17-24.

40. Shi Q, Presutti R, Selchen D, Saposnik G. Delirium in acute stroke: a systematic review and meta-analysis. Stroke. 2012;43:645-9.

41. Schuiling WJ, Rinkel GJ, Walchenbach R, de Weerd AW. Disorders of sleep and wake in patients after subarachnoid hemorrhage. Stroke. 2005;36:578-82.

42. Meng $\mathrm{H}$, Liu T, Borjigin J, Wang MM. Ischemic stroke destabilizes circadian rhythms. J Circadian Rhythms. 2008;6:9.
43. Kawada K, Ohta T, Tanaka K, Miyamura M, Tanaka S. Addition of suvorexant to ramelteon therapy for improved sleep quality with reduced delirium risk in acute stroke patients. J Stroke Cerebrovasc Dis. 2019;28:142-8.

44. Kilic U, Yilmaz B, Ugur M, Yuksel A, Reiter RJ, Hermann DM, et al. Evidence that membrane-bound G protein-coupled melatonin receptors MT1 and MT2 are not involved in the neuroprotective effects of melatonin in focal cerebral ischemia. J Pineal Res. 2012;52:228-35.

45. Chern CM, Liao JF, Wang YH, Shen YC. Melatonin ameliorates neural function by promoting endogenous neurogenesis through the MT2 melatonin receptor in ischemic-stroke mice. Free Radic Bio Med. 2012;52:1634-47.

46. Dubocovich ML, Yun K, Al-Ghoul WM, Benloucif S, Masana MI. Selective MT2 melatonin receptor antagonists block melatonin-mediated phase advances of circadian rhythms. FASEB J. 1998;12:1211-20.

47. Marion P, Vadot L, Estanove JF, Lapeyre D, Estanove S, George M. Cardiac assistance and substitutes for cardiocirculatory function. J Cardiovasc Surg (Torino). 1971;12:218-22.

48. Yu S, Wang $X$, Geng $P$, Tang $X$, Xiang $L$, Lu X, et al. Melatonin regulates PARP1 to control the senescence-associated secretory phenotype (SASP) in human fetal lung fibroblast cells. J Pineal Res. 2017;63:e12405.

49. Pei Z, Pang SF, Cheung RT. Pretreatment with melatonin reduces volume of cerebral infarction in a rat middle cerebral artery occlusion stroke model. J Pineal Res. 2002;32:168-72.

50. Pei Z, Pang SF, Cheung RT. Administration of melatonin after onset of ischemia reduces the volume of cerebral infarction in a rat middle cerebral artery occlusion stroke model. Stroke. 2003;34:770-5.

51. Robertson NJ, Martinello K, Lingam I, Avdic-Belltheus A, Meehan C, AlonsoAlconada $\mathrm{D}$, et al. Melatonin as an adjunct to therapeutic hypothermia in a piglet model of neonatal encephalopathy: a translational study. Neurobiol Dis. 2019;121:240-51.

52. Greenblatt DJ, Harmatz JS, Karim A. Age and gender effects on the pharmacokinetics and pharmacodynamics of ramelteon, a hypnotic agent acting via melatonin receptors MT1 and MT2. J Clin Pharmacol. 2007;47:485-96.

53. Harpsoe NG, Andersen LP, Gogenur I, Rosenberg J. Clinical pharmacokinetics of melatonin: a systematic review. Eur J Clin Pharmacol. 2015;71:901-9.

54. Koike M, Shibata M, Tadakoshi M, Gotoh K, Komatsu M, Waguri S, et al. Inhibition of autophagy prevents hippocampal pyramidal neuron death after hypoxicischemic injury. Am J Pathol. 2008;172:454-69.

55. Wen YD, Sheng R, Zhang LS, Han R, Zhang $X$, Zhang XD, et al. Neuronal injury in rat model of permanent focal cerebral ischemia is associated with activation of autophagic and lysosomal pathways. Autophagy. 2008;4:762-9.

56. Fu L, Huang L, Cao C, Yin Q, Liu J. Inhibition of AMP-activated protein kinase alleviates focal cerebral ischemia injury in mice: Interference with mTOR and autophagy. Brain Res. 2016;1650:103-11.

57. Hadley G, Beard DJ, Couch Y, Neuhaus AA, Adriaanse BA, DeLuca GC, et al. Rapamycin in ischemic stroke: Old drug, new tricks? J Cereb Blood Flow Metab. 2019;39:20-35.

58. Wang S, Xue H, Xu Y, Niu J, Zhao P. Sevoflurane postconditioning inhibits autophagy through activation of the extracellular signal-regulated kinase cascade, alleviating hypoxic-ischemic brain injury in neonatal rats. Neurochem Res. 2019;44:347-56.

59. Tao J, Shen C, Sun Y, Chen W, Yan G. Neuroprotective effects of pinocembrin on ischemia/reperfusion-induced brain injury by inhibiting autophagy. Biomed Pharmacother. 2018:106:1003-10.

60. Zheng Y, Hou J, Liu J, Yao M, Li L, Zhang B, et al. Inhibition of autophagy contributes to melatonin-mediated neuroprotection against transient focal cerebral ischemia in rats. J Pharmacol Sci. 2014;124:354-64.

61. Chen WR, Liu HB, Chen YD, Sha Y, Ma Q, Zhu PJ, et al. Melatonin attenuates myocardial ischemia/reperfusion injury by inhibiting autophagy via an AMPK/ mTOR signaling pathway. Cell Physiol Biochem. 2018;47:2067-76.

62. Han F, Chen YX, Lu YM, Huang JY, Zhang GS, Tao RR, et al. Regulation of the ischemia-induced autophagy-lysosome processes by nitrosative stress in endothelial cells. J Pineal Res. 2011:51:124-35. 